\title{
Broadcast in 5G Wireless Communication Networks and Beyond: An Overview of Public Safety Communications
}

\author{
Pimmy Gandotra ${ }^{1+}$, Vimal Bhatia ${ }^{2}$ and Brejesh Lall ${ }^{1}$ \\ ${ }^{1}$ Indian Institute of Technology, Delhi \\ ${ }^{2}$ Indian Institute of Technology, Indore
}

\begin{abstract}
The objective of this paper is to cover the present and future scope of the public safety communication, which targets timely and accurate delivery of critical information. The evolution of these services over the generations of wireless communication networks (WCNs) has been discussed in this paper. The basic broadcasting mechanism, for the dissemination of essential information during emergency and lockdown has been described, followed by the supporting architectures for information broadcasting. The role of various technologies of the $5 \mathrm{G}$ WCNs, and beyond, in improving the efficiency of the functioning of these networks has been stated. The technologies include spectrum sharing, network slicing, software defined networking (SDN), augmented reality (AR) and virtual reality (VR). A seamless integration of these technologies can help in achieving high efficiency, optimal resource and power availability. Challenges in executing these are also discussed.
\end{abstract}

Keywords: 5G and Beyond 5G, IoT, Spectrum Sharing, AR, VR, SDN, broadcast, public warning.

\section{Introduction}

The 5G WCNs are expected to comprise of multi-radio systems, levelled upon various interfaces, supporting high throughput and low latency. These come along with convergence of many radio access technologies (RATs), including WiFi, LTE and 5G New Radio (NR) as a result of the advancements in the technologies over the past decades. All these play a key role in the establishment of critical/public safety communications. These mainly target provisioning quality service to people after disasters/calamities that may be natural or man-made. As a result, the ICT plays an important role in the operation of emergency services and have tremendous potential to deal with uncertain events like riots, pandemics, lockdowns etc.

For the purpose of sending alerts to numerous people within the same area under emergency, a telecom broadcast is an appropriate method. It was first introduced by $3 \mathrm{GPP}$ and termed as multimedia broadcast/multicast service (MBMS) in Release 6. It evolved to enhanced eMBMS (eMBMS) in the 3GPP Release 11, and to further enhanced MBMS (FeMBMS) in Release 14. To provide an all-inclusive solution for broadcast and/or multicast in the 5G WCNs and beyond, 5G Xcast has been taken up under the H2020 Phase II of 5GPPP project [1]. The broadcast nature of the 5G and beyond WCNs is essential in the evolving communication era, which is continuously seeking seamless integrity to meet the diverse subscriber demands. For instance, fire instances in a building, breakout of a deadly diseases like the COVID-19, and other situations, where public safety communications play a key role.

With the rising mandate in public safety communication, the role of ICT in providing these services has gained significant momentum. 3GPP, in its Release 13, has introduced the Isolated E-UTRAN Operation for Public Safety (IOPS) [2]. The IOPS provides connectivity to the users even in the absence of availability of connection of the BS to the core. Push-to-talk was also part of 3GPP Release 13, and the data and video were part of Release 14. Enhancements in the public safety services have been carried out in Release 15. In the US, the First Net project has been initiated public safety in coastal areas. Various countries have also initiated such projects. Most of the public safety communications initially exchanged emergency information through voice only. However, with the advancements in the cellular technology, information exchange via data and video has been made possible. Standardization agencies are working towards the development of

\footnotetext{
+ Corresponding author. Tel.: + 919622290794.

E-mail address: pimmy.gandotra@gmail.com.
} 
standards for public safety like the Proximity services [3], group calls [4], and the like. The ITU-T has a special focus group for the disaster relief system.

\section{Broadcast and Public Safety Communication: An Overview of the Evolutionary Process}

The development of priority communication networks over the existing cellular networks, with optimal management of the QoS and demand priorities is studied in [5]. Currently although not standardized, broadcast in 5G and beyond WCNs is mentioned in terms of Point-to-multipoint (PTM) communication, as is discussed in [6]. The differences between point-to-point (PTP) and PTM are drawn in Table 1. Popular examples of broadcasting include streaming of an ongoing match in a stadium, popular content caching etc [7]. Using unicast for such scenarios would result in overloading and network congestion, along with a longer execution time. Broadcasting comes with an additional advantage of higher resource efficiency [6].

Table 1: PTP and PTM Transmissions: A Comparative Analysis

\begin{tabular}{|l|l|l|}
\hline Feature & Point-to-point (PTP) transmission & Point-to-multipoint (PTM) transmission \\
\hline Supported modes & AM or UM & UM only \\
\hline Protocol stack support & PHY, MAC, PLC, PDCP, SDAP & PHY,MAC, RLC, PDCP (Transparent) \\
\hline Standards & 3GPP Release 15 & $\begin{array}{l}\text { Not standardized yet (Expected to be part of } \\
\text { 3GPP Release 16 and 17) }\end{array}$ \\
\hline Re-transmission scheme & ARQ & CQI based re-transmissions \\
\hline Error correction schemes & $\begin{array}{l}\text { Low density parity check (LDPC) } \\
\text { and polar codes }\end{array}$ & Raptor codes \\
\hline
\end{tabular}

The traditional public safety services involved the use of professional mobile radio (PMR). The PMR networks are mostly privately owned and were a group of narrowband services, with data rates ranging from about $9.6 \mathrm{kbps}$ to a few hundreds of kbps [5]. These data rates are insufficient for the broadband technologies of the present. In order to emulate with the advancements in technology, the safety networks need to have a close inclination with the commercial cellular networks. Thus, the trend is heading towards the use of commercial mobile networks for provision of the public safety. A comparison between PMR and LTE has been drawn in Table 2.

The notion of broadcast/multicast was first introduced by 3GPP for optimal distribution of traffic, in case of large user count, and was termed as MBMS. It evolved to eMBMS in the 3GPP Release 11, supporting traffic offloading. The advancements in eMBMS have been continuing, with the introduction of MBMS on demand (MOOD) supporting a higher dynamicity in the network. With the inclusion of novel frame formats, the further enhanced MBMS (FeMBMS) has been introduced by 3GPP. The transitions in these standards are illustrated in Table 3.

Table 2: Comparison Between PMR and LTE

\begin{tabular}{|l|l|l|}
\hline Features & PMR & LTE \\
\hline Application support & $\begin{array}{l}\text { Mostly voice calling, deploy push-to- } \\
\text { talk technology }\end{array}$ & Mostly Data Applications \\
\hline Support for group call & Yes & No \\
\hline Latency & Low & High \\
\hline Push to talk support & Yes & No \\
\hline Standards & TETRA, P25, iDEN & 3GPP Release 9 \\
\hline Network Type & Private Networks & Public, MNO operated \\
\hline Bandwidth occupancy & Narrowband & Broadband \\
\hline Security & $\begin{array}{l}\text { E2E encryption, from device to network } \\
\text { and back to device }\end{array}$ & $\begin{array}{l}\text { Encryption at base station, thus less robust } \\
\text { security }\end{array}$ \\
\hline Permissible Power Levels & Higher power output & Restricted power output \\
\hline
\end{tabular}




\section{Architectures for Broadcast in Cellular Networks}

An illustration of broadcasting of information is depicted in Fig. 1, where the data as well as control information is transmitted to the gateway by the broadcast/multicast service center (BM-SC). The BM-SC controls the MBMS gateway and performs traffic mapping onto broadcast bearers.

Table 3: Evolving Broadcasting Technologies

\begin{tabular}{|l|l|l|l|}
\hline Parameters & MBMS & eMBMS & FeMBMS \\
\hline Maximum data rate support & $256 \mathrm{Kbps}$ & $36 \mathrm{Mbps}$ to 48 Mbps & $100 \%$ cell capacity \\
\hline Power consumption & Very low & Higher & High \\
\hline 3GPP Release & Release 9 & Release 12 & Release 14 \\
\hline Subcarrier Spacing & $15 \mathrm{KHz}$ & $7.5 \mathrm{KHz}, 1.25 \mathrm{KHz}$ & $\begin{array}{l}\text { Four different configurations } \\
\text { supported; Legacy LTE, MBMS } \\
\text { and eMBMS }\end{array}$ \\
\hline Cyclic prefix & $16.6 \mu \mathrm{s}$ & $33 \mu \mathrm{s}$ & $200 \mu \mathrm{s}$ \\
\hline Inter-site Distance & $5 \mathrm{~km}$ & $10 \mathrm{~km}$ & Greater than 15km \\
\hline
\end{tabular}

Use of public safety systems and broadcasting for the dissemination of emergency messages has had a significant impact on the day-to-day lives of everyone. Initial systems were introduced by Motorola, and date back to 1930s. Numerous systems have been used by the emergency service providers over the years [8], successfully reducing crime rates and creating awareness among folks. The public safety communication over the cellular networks may or may not have the infrastructure availability. As a result, infrastructurebased, and infrastructure-less set ups are proposed, which are as discussed next.

\subsection{Infrastructure-based Architecture}

A set-up for the public safety communication, under the availability of WCNs is available, depicted in Fig. 2. The architecture is a distributed set-up, with server and core networks placed at different locations. The various components and interfaces are explained in [7].

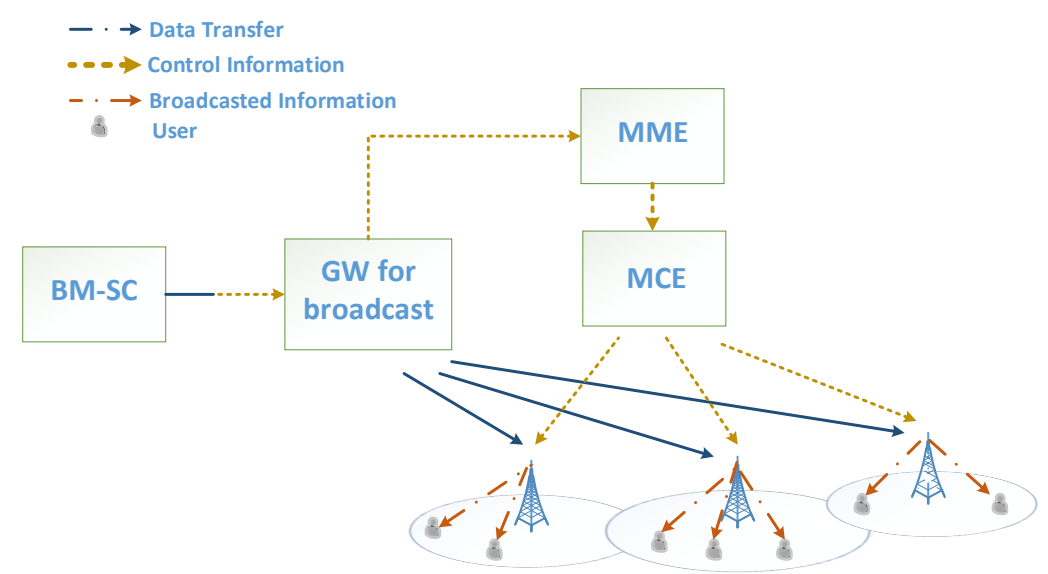

Fig. 1: Illustrating the broadcast of information

\subsection{Infrastructure-less Architecture}

In case of unavailability of the commercial networks, rapidly deployable networks (RDNs) are proposed. These support critical voice and data services. The provision of connecting drones, cameras and sensors to these networks add advantage to the set up. Lite-EPCs emulate the functions of a commercial EPC in an LTE network. The information regarding spectrum for the RDN can be obtained via the LSA system. The decision on sharing of spectral resources is taken by the distributed LSA controllers. An RDN set-up is illustrated in [6], and illustrated in Fig. 3. In case of loss of infrastructure, the unmanned aerial vehicles can route the information about the emergency site to the rescue workers. Both the architecture types, discussed in this section, can be put to use for public safety communications in $5 \mathrm{G}$ and beyond WCNs. 


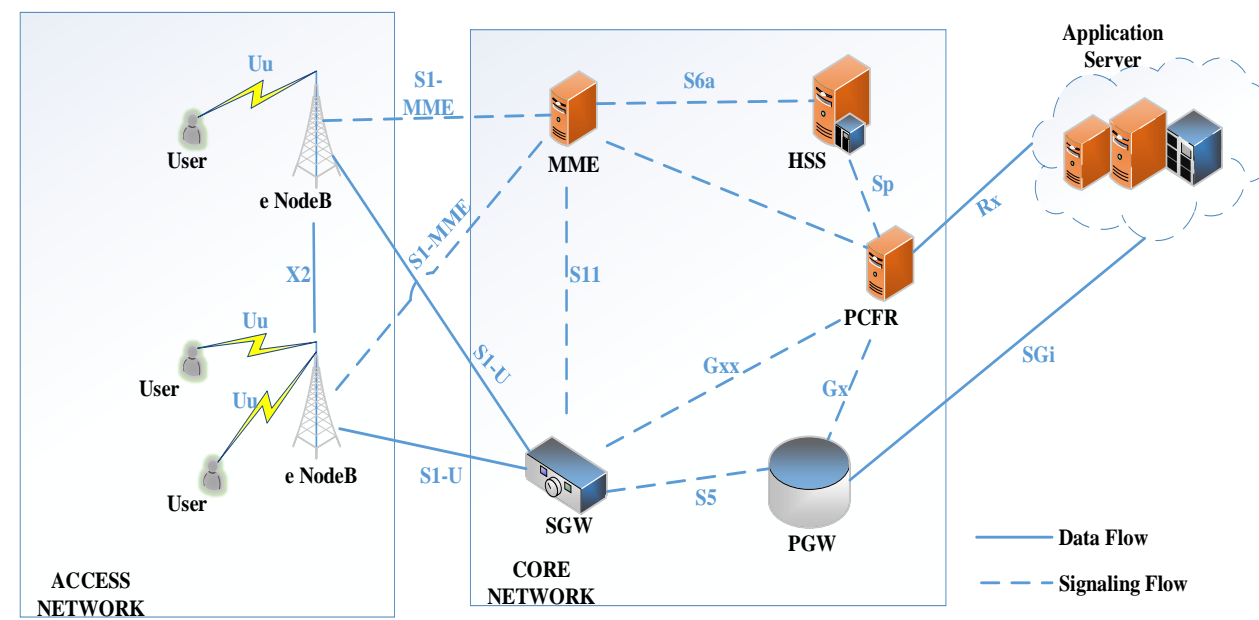

Fig. 2: Infrastructure based architecture for Public Safety Communication

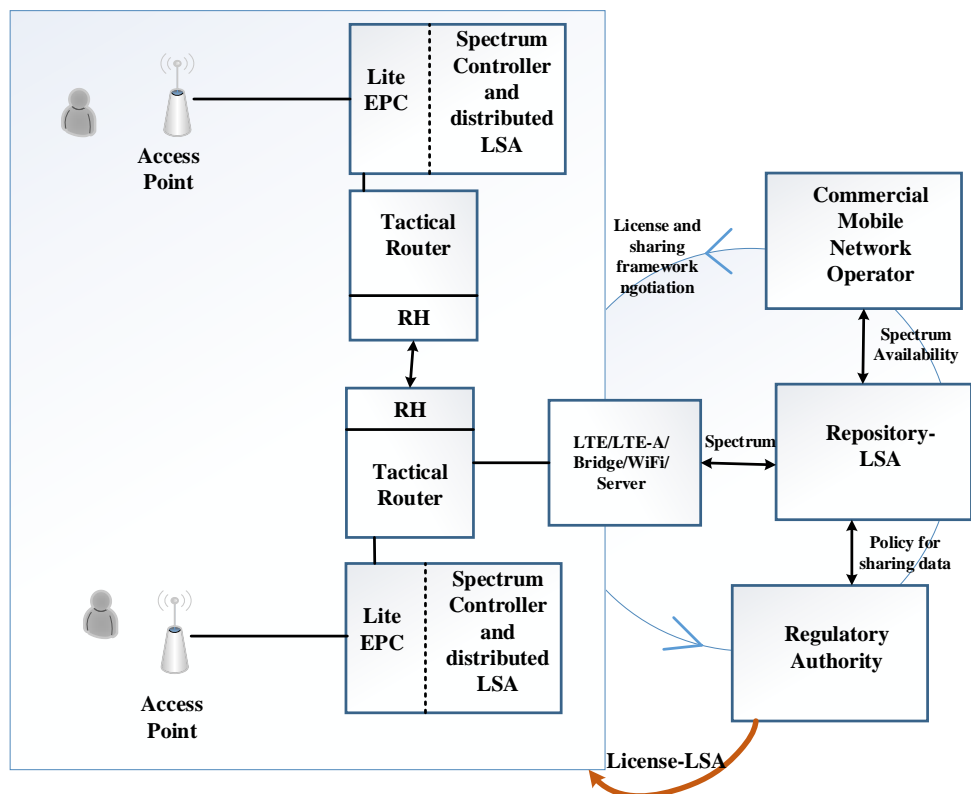

Fig. 3: Rapidly Deployable network Architecture, using LSA

\section{Public Safety Communications in 5G Networks and Beyond: The Technological Advancement}

As the development of $5 \mathrm{G}$ WCNs is associated with numerous technologies for supporting various applications in different areas, these technologies shall play a key role in public safety network set up and communication establishment. Some of these have been discussed in this section, and depicted in Fig. 4.

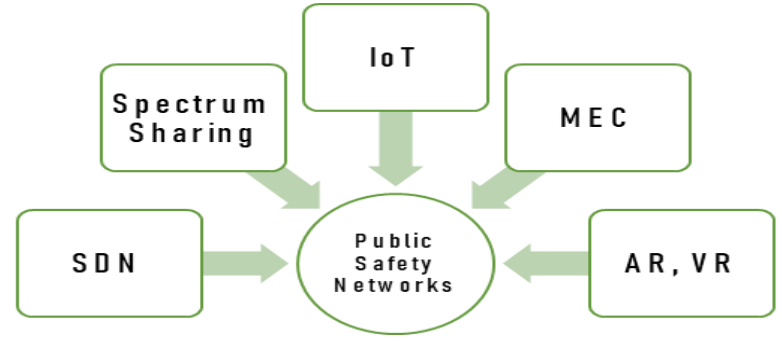

Fig. 4: Key Technologies for Efficient Public Safety Communication

\subsection{Software-Defined Networking (SDN) and Network Slicing}

It allows operators to customize the networks as per the service requirements. SDN supports creation of a logical network within the existing physical networks, so as to guarantee optimal resources [9]. Examples include ambulance communication, Network embedded online disaster (NEOD) [10]. Another reliable 
method of providing services in emergency situations is the decentralize SDN (D-SDN). SDN can also prove to optimize the energy consumption levels in the network [11], supporting a green environment.

\subsection{Spectrum Sharing}

The spectrum sharing technology allows better and more spectral resources to be used for the public safety communication, maintaining the requisite QoS in the WCNs. Optimal resource availability for the safety communication reduces the chances of migration of the communication to other bands. The massive potential of spectrum sharing in the public safety communications has been elaborated in [12].

\subsection{Internet of Things}

In case of an emergency situation, device compatibility to coordinate is essential which is largely supported by the IoT. The enormous generated data can help in the situation analysis, thereby effectively combating the situation. The concept of smart community sends alerts to its subscribers, during any suspicious movements in and around their homes and/or workplaces, with the help of the smart phone apps. Various intelligent disaster handling mechanisms have been proposed in literature, as described in [13], improving the efficacy and predictability.

\subsection{Multi-access Edge Computing}

As MEC allows a real-time access with an ultra-low latency, it can act as a complementing technology. Its use shall be particularly useful in the Vehicle-to-vehicle (V2V) communications, real-time video analytics and optimal traffic and energy management [14]. As the content to be broadcasted requires streaming and formatting, the MEC server can do these and provide a quasi-real-time video. The resource usage can also be finely tuned with MEC.

\subsection{Augmented and Virtual Reality (AR and VR)}

Smart phones, equipped with the AR mechanism enable surveying of situations and recreating the environment. This eventually helps the people to be prepared in advance, for uncertainties [15]. The key points of interest can be identified beforehand and a stock of the possible hazards can be obtained. This has gained popularity in the police training. Potential hide-outs of the terrorists can be identified and mis happenings can be avoided.

Knowing an overview of the potential $5 \mathrm{G}$ technologies, it can be deduced that these technologies can provide an unobstructed and efficient broadcasting in the 5G and beyond WCNs. Other contributing technologies shall be device-to-device (D2D) communication, visible light communication (VLC), etc. The backhaul technology used will also play a key role in such an information broadcast, in 5G and beyond WCNs.

\section{Research Challenges}

Several advancements are underway for providing broadcast efficiently, however, it suffers from a number of challenges. In case of calamities, numerous subscribers need access to the network which can result in a severe network congestion and scarcity of the available spectrum.

The public safety service providers find it difficult to obtain the financial support for their network set ups. Security is another major concern in such a scenario. It is because the relevant information is broadcasted to several people simultaneously overloads the network, making security a major concern. Along with an overloaded network comes a large amount of signaling too.

As $5 \mathrm{G}$ comes with the ultra-dense network deployment, prioritization of information to be sent is essential. The specific area, where the necessary information must be broadcasted, should be first identified and information should be sent on priority basis. Clearly, significant challenges still exist in the road to a complete next generation enabled public safety communication [16].

\section{Conclusion}

This paper presented a study on public safety communication in the $5 \mathrm{G}$ and beyond WCNs. The various studies carried out for public safety and its progression in terms of broadcasting standards has been stated. 
The supporting architectures include infrastructure based and infrastructure less layouts, and are stated. The unfolding generations of the WCNs seek an unparallel evolution in the public safety communication networks as well. For the same, the potential of the wireless technologies is studied for the emergency networks. However, these need redressal of some key challenges, which have also been stated.

\section{References}

[1] Available at: $5 \mathrm{~g}$-xcast.eu.

[2] 3gpp TS 22.346,"'Technical Specification Group Services and System Aspects; Isolated -UTRAN Operation for Public Safety, Stag 1 (Rel 13),” Sept 2014.

[3] 3GPP TS 23.303, version 15.1.0 Release 15, "Universal Mobile Telecommunication Services (UMTS); LTE; Proximity-based services (ProSe); Stage 2", 2018.

[4] Group Communication System Enablers for LTE (GCSE_LTE), 3GPP TS 22.468 version 12.1.0 Release 12, 2014.

[5] Höyhtyä, Marko, et al. "Critical communications over mobile operators' networks: $5 \mathrm{G}$ use cases enabled by licensed spectrum sharing, network slicing and QoS control." IEEE Access, vol. 6, pp 73572-73582, 2018.

[6] Säily, Mikko, et al. "Enabling Efficient Point-to-Multipoint Transmissions in 5G RAN.", IEEE Vehicular Technology Magazine, 2019.

[7] Lecompte, David, and Frédéric Gabin. "Evolved multimedia broadcast/multicast service (eMBMS) in LTEadvanced: overview and Rel-11 enhancements." IEEE Communications Magazine, vol. 50, no. 11, pp. 68-74, 2012.

[8] “The future is now: Public Safety LTE Communications," Motorola white paper, 2012.

[9] Study on Management and Orchestration of Network Slicing for Next Generation Network (Release 15), document TR 28.801 V1.2.0, 3GPP, May 2017.

[10] Wetterwald, Michelle, et al. "SDN for public safety networks." HAL, 2016.

[11] Usman, Muhammad, et al. "A software-defined device-to-device communication architecture for public safety applications in 5G networks." IEEE Access, vol. 3, pp. 1649-1654, 2015.

[12] Yuksel, Murat, et al. "Pervasive spectrum sharing for public safety communications." IEEE Communications Magazine, vol. 54, no.3, pp. 22-29, 2016.

[13] Câmara, Daniel, and Navid Nikaein, eds. Wireless public safety networks 2: a systematic approach. Elsevier, 2016.

[14] Merwaday, Arvind, and Ismail Guvenc. "UAV assisted heterogeneous networks for public safety communications."EEE wireless communications and networking conference workshops (WCNCW), 2015.

[15] Lutz, Robyn R. "Safe-AR: Reducing risk while augmenting reality." IEEE 29th International Symposium on Software Reliability Engineering (ISSRE), 2018.

[16] Ulema, Mehmet. Fundamentals of Public Safety Networks and Critical Communications Systems: Technologies, Deployment, and Management. John Wiley \& Sons, 2019. 